ANNALES

POLONICI MATHEMATICI

LXXVIII.2 (2002)

\title{
Nontaut foliations and isoperimetric constants
}

\author{
by KONRAD BLACHOWSKI (Łódź)
}

\begin{abstract}
We study nontaut codimension one foliations on closed Riemannian manifolds. We find an estimate of some constant derived from the mean curvature function of the leaves of a foliation by some isoperimetric constant of the manifold. Moreover, for foliated 2-tori and the 3-dimensional unit sphere, we find the infimum of the former constants for all nontaut codimension one foliations.
\end{abstract}

1. Introduction. In this paper we study nontaut codimension one foliations on closed manifolds. A foliation is said to be taut if there exists a Riemannian metric on the manifold in which all leaves of the foliation are minimal submanifolds. For codimension one foliations there are many conditions equivalent to tautness. One of them is that there exists a transverse loop which meets all leaves of the foliation.

G. Reeb [Re] was the first to find a connection between the volume form of leaves of a codimension one foliation, the mean curvature function of the leaves and the volume form of the whole manifold. After almost thirty years $\mathrm{H}$. Rummler $[\mathrm{Ru}]$ showed an analogous relation for any codimension (so-called Rummler formula).

Let $\mathcal{F}$ be an oriented codimension one foliation on a closed Riemannian manifold $M$ of dimension $n+1$. Let $H_{\mathcal{F}}(x)$ be the mean curvature of the leaf through $x$ at the point $x$. The function $H_{\mathcal{F}}: M \rightarrow \mathbb{R}$ is smooth. We put $I(\mathcal{F})=\int_{M}\left|H_{\mathcal{F}}\right|^{n+1} \Omega$, where $\Omega$ is the volume form of $M$.

Recently, G. I. Oshikiri [Os] has shown, using the Rummler formula, that $I(\mathcal{F}) \geq I(M)$ for any nontaut codimension one foliation $\mathcal{F}$. Here, $I(M)$ is the isoperimetric constant of $M$. One can ask if this estimate is best possible. We will show that $I(\mathcal{F})>\left(k_{\mathcal{F}}-1\right) \cdot I(M)$, where $k_{\mathcal{F}} \geq 2$ is an integer depending on $\mathcal{F}$. Moreover, if $M$ is a 2-dimensional torus or 3-dimensional unit sphere, then $I(\mathcal{F})>2 \cdot I(M)$.

2000 Mathematics Subject Classification: Primary 53C12.

Key words and phrases: foliation, taut, mean curvature, isoperimetric constant, slanted torus. 
Another question we can ask is: what is the infimum of $I(\mathcal{F})$ for all nontaut codimension one foliations $\mathcal{F}$ on $M$ ? The answer is given for foliations on 2-tori and the 3-dimensional unit sphere. In the case of foliations on the sphere $S^{3}$, the infimum found suggests that an isoperimetric constant coming from separations of $S^{3}$ by tori is achieved by the Clifford torus. This statement seems to be close to the so-called Lawson conjecture about minimal tori on $S^{3}$.

\section{Preliminaries}

2.1. Foliations. Let $(M, g)$ be a smooth oriented closed Riemannian $n$ manifold and let $\nabla$ be the Levi-Civita connection on $(M, g)$. We denote by $\mathscr{M}$ the family of all closed $(n-1)$-submanifolds $S$ that separate $M$ into two connected open submanifolds $M_{1}$ and $M_{2}$ satisfying $M \backslash S=M_{1} \cup M_{2}$ and $\partial M_{1}=\partial M_{2}=S$. Then we can define the isoperimetric constant $I(M)$ by

$$
I(M)=\inf _{S \in \mathscr{M}} \frac{\left(\operatorname{Vol}_{n-1}(S)\right)^{n}}{\min \left\{\operatorname{Vol}_{n}\left(M_{1}\right), \operatorname{Vol}_{n}\left(M_{2}\right)\right\}^{n-1}},
$$

where $\operatorname{Vol}_{n-1}(S)$ is the $(n-1)$-volume of $S$, and $\operatorname{Vol}_{n}\left(M_{1}\right)$ is the $n$-volume of $M_{1}$. It is known that $I(M)>0$ (see [Ch]).

Let $\mathcal{F}$ be a smooth transversely oriented codimension one foliation on $M$. Let $x \in M$ and $U$ be an open neighbourhood of $x$ such that there exist vector fields $E_{1}, \ldots, E_{n-1}$ on $U$ spanning $\left.T \mathcal{F}\right|_{U}$. We can assume that $g\left(E_{i}, E_{j}\right)=\delta_{i}^{j}$ for $i, j=1, \ldots, n-1$. Moreover, let $N$ be a unit vector field on $U$ orthogonal to $\mathcal{F}$. We assume that for any $y \in U$ the system $\left\{E_{1}(y), \ldots, E_{n-1}(y)\right\}$ is a positively oriented frame of $T_{y} L_{y}$ and $\left\{N(y), E_{1}(y), \ldots, E_{n-1}(y)\right\}$ is a positively oriented basis of $T_{y} M$.

Definition 2.1. Under the above assumptions we locally define the function $H_{\mathcal{F}}$ by

$$
H_{\mathcal{F}}=\sum_{i=1}^{p} g\left(\nabla_{E_{i}} E_{i}, N\right)
$$

and we call it the mean curvature function of the leaves of $\mathcal{F}$.

Now let $X_{i} \in \mathfrak{X}(M)$ for $i=1, \ldots, n-1$ and

$$
\chi_{\mathcal{F}}\left(X_{1}, \ldots, X_{n-1}\right)=\operatorname{det}\left[g\left(E_{i}, X_{j}\right)\right]_{i, j=1, \ldots, n-1} .
$$

Then $\chi_{\mathcal{F}}$ is a well defined $(n-1)$-form on $M$ and $\left.\chi_{\mathcal{F}}\right|_{L}$ is the volume form of a leaf $L \in \mathcal{F}$. Let $\Omega$ be the volume form of $M$. We put

$$
I(\mathcal{F})=\int_{M}\left|H_{\mathcal{F}}\right|^{n} \Omega
$$

Under the above notations we have the Rummler formula [Ru]

$$
d \chi_{\mathcal{F}}=-H_{\mathcal{F}} \cdot \Omega \text {. }
$$




\subsection{Nontaut codimension one foliations}

Definition 2.2. A foliation $\mathcal{F}$ on an oriented compact manifold $M$ is said to be taut if there exists a Riemannian metric on $M$ such that all the leaves of $\mathcal{F}$ are minimal submanifolds of $M\left(H_{\mathcal{F}} \equiv 0\right)$.

According to Corollary 3 from [Su, p. 220], an oriented codimension one foliation is taut iff every leaf meets a closed curve transverse to the foliation. We say that a nonempty compact subset $D \subset M$ is a positively (negatively) foliated domain iff $D$ is a union of leaves of $\mathcal{F}$ and the unit normal field points outward (resp. inward) everywhere on the boundary $\partial D$. Note that $\partial D$ is a finite union of compact leaves. Oshikiri has proved the following

THEOREM 2.3 ([Os] $]$ ). A transversely oriented codimension one foliation on an oriented compact manifold is taut if and only if it does not contain any positively or negatively foliated domain.

Let $\mathcal{F}$ be a nontaut transversely oriented codimension one foliation on a compact oriented manifold $M$. Then, by Theorem 2.3, the family $\mathscr{D}_{+}$(resp. $\mathscr{D}_{-}$) of positively (resp. negatively) foliated domains is nonempty. It is easy to see that for $D_{1}, D_{2} \in \mathscr{D}_{+}$(resp. $D_{1}, D_{2} \in \mathscr{D}_{-}$) we have either $D_{1} \cap D_{2}=\emptyset$ or $D_{1} \cap D_{2} \in \mathscr{D}_{+}$(resp. $D_{1} \cap D_{2} \in \mathscr{D}_{-}$). Denote by $\mathscr{D}_{+}^{0}$ (resp. $\mathscr{D}_{-}^{0}$ ) the family of all minimal positively (resp. negatively) foliated domains. Then a simple argument leads to the following lemma.

Lemma 2.4. The set $\mathscr{D}^{0}=\mathscr{D}_{+}^{0} \cup \mathscr{D}_{-}^{0}$ is finite and

$$
k_{\mathcal{F}}=\operatorname{card}\left(\mathscr{D}^{0}\right) \geq 2 \text {. }
$$

2.3. Total curvature of a codimension one foliation of $S^{n}$. Suppose that $M$ is an open subset of the unit sphere $S^{n}$ and that $\mathcal{F}$ is an oriented codimension one foliation on $M$. We denote by $N$ a unit vector field on $S^{n}$ normal to $\mathcal{F}$. Let $K_{\mathcal{F}}(x)$ be the Gauss-Kronecker curvature of $L_{x} \subset S^{n}$ at the point $x$ and let $\mathcal{S}(n, n-1)$ be the family of all complete totally geodesic $(n-1)$-submanifolds of $S^{n}$, that is, of great spheres $S=H \cap S^{n}$, where $H$ is an $n$-dimensional vector subspace of $\mathbb{R}^{n+1}$. The set $\mathcal{S}(n, n-1)$ has a natural structure of a smooth differentiable manifold and has a unique measure $\mu_{\mathcal{S}}$ invariant under the action of the group of isometries of $S^{n}$ such that $\mu_{\mathcal{S}}(\mathcal{S}(n, n-1))=\frac{1}{2} \cdot \operatorname{Vol} S^{n}$.

Let $S \in \mathcal{S}(n, n-1)$. We denote by $\left.\mathcal{F}\right|_{S}$ the family of all connected components of intersections $L \cap S$, where $L \in \mathcal{F}$. Then for almost all $S \in \mathcal{S}(n, n-1)$, $\mathcal{F}_{S}$ is a codimension one foliation on $S$ with only isolated singularities and the orthogonal projection $N_{S}$ of the vector field $N$ onto $T S$ is a tangent vector field on $S$ with only isolated singularities. Moreover the foliation $\left.\mathcal{F}\right|_{S}$ has a singularity at $x \in S$ iff $S$ is tangent to $\mathcal{F}$ at $x$. We denote by $\Sigma\left(\left.\mathcal{F}\right|_{S}\right)$ the set of all singular points of $\left.\mathcal{F}\right|_{S}$. Clearly, if $x \in S \backslash \Sigma\left(\left.\mathcal{F}\right|_{S}\right)$ then the 
vector $N_{S}(x)$ is orthogonal to the foliation $\mathcal{F}_{S}$. Suppose $\Sigma\left(\left.\mathcal{F}\right|_{S}\right)$ consists of isolated points and let $x \in \Sigma\left(\left.\mathcal{F}\right|_{S}\right)$. We define the sign of a singular point $x$ of $\mathcal{F}_{S}$ as follows:

$$
\operatorname{sign}(x)=i(x)
$$

where $i(x)$ is the index of the vector field $N_{S}$ at $x$. Langevin [La] shows that for almost all $S \in \mathcal{S}(n, n-1)$ such that $\Sigma\left(\left.\mathcal{F}\right|_{S}\right)$ consists of isolated points we have $|i(x)|=1$ for $x \in \Sigma\left(\left.\mathcal{F}\right|_{S}\right)$. We denote by $\widetilde{\mathcal{S}}$ the subset of $\mathcal{S}(n, n-1)$ of all $S$ such that $\Sigma\left(\left.\mathcal{F}\right|_{S}\right)$ consists of isolated points and $|i(x)|=1$ for all $x \in \Sigma\left(\left.\mathcal{F}\right|_{S}\right)$.

Definition 2.5. For $S \in \widetilde{\mathcal{S}}$ we put

$$
|\mu|(\mathcal{F}, S)=\operatorname{card}\left(\Sigma\left(\left.\mathcal{F}\right|_{S}\right)\right)
$$

and if $|\mu|(\mathcal{F}, S)<\aleph_{0}$, then

$$
\mu(\mathcal{F}, S)=\sum_{x \in \Sigma\left(\left.\mathcal{F}\right|_{S}\right)} \operatorname{sign}(x) .
$$

Under the above assumptions the following theorem holds.

Theorem 2.6 ([La1], [LaLe], [La]).

$$
\int_{M}\left|K_{\mathcal{F}}\right| \Omega=\int_{\mathcal{S}(n, n-1)}|\mu|(\mathcal{F}, S) d \mu_{\mathcal{S}}(S) .
$$

Moreover

$$
\int_{M} K_{\mathcal{F}} \Omega=\int_{\mathcal{S}(n, n-1)} \mu(\mathcal{F}, S) d \mu_{\mathcal{S}}(S) .
$$

Now let $\mathcal{F}$ be a codimension one foliation on the sphere $S^{3}$. We suppose that $S \in \widetilde{\mathcal{S}} \subset \mathcal{S}(3,2)$. By the Poincaré-Hopf theorem (see [Ho]) we have

$$
\sum_{x \in \Sigma\left(\left.\mathcal{F}\right|_{S}\right)} \operatorname{sign}(x)=\chi(S)=2,
$$

where $\chi(S)$ is the Euler characteristic of $S$. Therefore $|\mu|(\mathcal{F}, S) \geq 2$ and $\mu(\mathcal{F}, S)=2$. As a simple corollary of the last theorem we obtain the following two theorems.

Theorem 2.7 ([La1], [La2], [La]). Let $\mathcal{F}$ be an oriented codimension one foliation on the 3 -sphere $S^{3}$. Then

$$
\int_{S^{3}}\left|K_{\mathcal{F}}\right| \Omega \geq 2 \pi^{2}
$$

ThEOREM 2.8 ([La1], [La2], [La]). Under the assumptions of 2.7 we have

$$
\int_{S^{3}} K_{\mathcal{F}} \Omega=2 \pi^{2} .
$$

We end this subsection with another theorem. 
Theorem 2.9 ([La1], [La2], [La]). For any oriented codimension one foliation $\mathcal{F}$ on the sphere $S^{3}$ we have

$$
\int_{S^{3}}\left|K_{\mathcal{F}}\right| \Omega>2 \pi^{2}
$$

\section{Results}

\subsection{The main inequality}

Theorem 3.1. Let $(M, g)$ be a closed Riemannian manifold of dimension $n$ and let $\mathcal{F}$ be a nontaut codimension one foliation on $M$. Then

$$
\int_{M}\left|H_{\mathcal{F}}\right|^{n+1} \Omega>\left(k_{\mathcal{F}}-1\right) I(M),
$$

where $\Omega$ is the volume form of $M$ and $H_{\mathcal{F}}$ is the mean curvature function of the leaves of $\mathcal{F}$.

Proof. Let $M=\bigcup_{i=1}^{k_{\mathcal{F}}} D_{i}$, where $D_{i}$ are positively or negatively foliated domains for $i=1, \ldots, k_{\mathcal{F}}$ such that $\operatorname{int} D_{i} \cap \operatorname{int} D_{j}=\emptyset$ for $i \neq j$. Then by the Stokes theorem, Rummler formula (5) and Hölder inequality, for all $i \in\left\{1, \ldots, k_{\mathcal{F}}\right\}$ we have

$$
\begin{aligned}
\text { Area } \partial D_{i} & =\left|\int_{\partial D_{i}} \chi_{\mathcal{F}}\right|=\left|\int_{D_{i}} d \chi_{\mathcal{F}}\right|=\left|\int_{D_{i}} H_{\mathcal{F}} \Omega\right| \leq \int_{D_{i}}\left|H_{\mathcal{F}}\right| \Omega \\
& \leq\left(\int_{D_{i}}\left|H_{\mathcal{F}}\right|^{n+1} \Omega\right)^{1 /(n+1)}\left(\int_{D_{i}} \Omega\right)^{n /(n+1)} \\
& =\left(\int_{D_{i}}\left|H_{\mathcal{F}}\right|^{n+1} \Omega\right)^{1 /(n+1)}\left(\operatorname{Vol} D_{i}\right)^{n /(n+1)}
\end{aligned}
$$

Therefore for all $i \in\left\{1, \ldots, k_{\mathcal{F}}\right\}$,

$$
\int_{D_{i}}\left|H_{\mathcal{F}}\right|^{n+1} \Omega \geq \frac{\left(\text { Area } \partial D_{i}\right)^{n+1}}{\left(\operatorname{Vol} D_{i}\right)^{n}} .
$$

Since there exists at most one $i_{0} \in\left\{1, \ldots, k_{\mathcal{F}}\right\}$ such that $\operatorname{Vol} D_{i_{0}}>\frac{1}{2} \operatorname{Vol} M$, from inequality (16) we obtain

$$
\begin{aligned}
\int_{M}\left|H_{\mathcal{F}}\right|^{n+1} \Omega & \geq \sum_{i \in \mathcal{I}} \frac{\left(\operatorname{Area} \partial D_{i}\right)^{n+1}}{\left(\operatorname{Vol} D_{i}\right)^{n}} \geq \frac{\left(\operatorname{Area} \partial D_{i_{0}}\right)^{n+1}}{\left(\operatorname{Vol} D_{i_{0}}\right)^{n}}+\sum_{i \in \mathcal{I} \backslash\left\{i_{0}\right\}} I(M) \\
& >\left(k_{\mathcal{F}}-1\right) I(M),
\end{aligned}
$$

where $\mathcal{I}=\left\{1, \ldots, k_{\mathcal{F}}\right\}$.

REMARK 3.2. The first inequality in (15) becomes an equality if the function $H_{\mathcal{F}}$ has a constant sign on $D_{i}$. The second inequality, by the Hölder theorem, can be replaced by an equality iff there exist nonvanishing constants $C_{1}$ and $C_{2}$ such that $C_{1}\left|H_{\mathcal{F}}\right|^{n+1}=C_{2}$, that is, iff $H_{\mathcal{F}}$ is a constant 
function on $D_{i}$. Hence, in order to get

$$
\int_{D_{i}}\left|H_{\mathcal{F}}\right|^{n+1} \Omega=I(M)
$$

the function $H_{\mathcal{F}}$ must be as above and $D_{i}$ has to be a subset of $M$ such that

$$
I(M)=\frac{\left(\text { Area } \partial D_{i}\right)^{n+1}}{\min \left\{\operatorname{Vol} D_{i}, \operatorname{Vol}\left(M \backslash \operatorname{int} D_{i}\right)\right\}^{n}} .
$$

REMARK 3.3. When all positively or negatively foliated domains $D_{i}$ are such that $\operatorname{Vol} D_{i} \leq \frac{1}{2} \operatorname{Vol} M$, the argument in the proof of Theorem 3.1 leads to

$$
\int_{M}\left|H_{\mathcal{F}}\right|^{n+1} \Omega \geq k_{\mathcal{F}} \cdot I(M)
$$

3.2. Slanted torus $T_{\alpha}(v, w)$. Let $v, w \in \mathbb{R}^{2}$ be linearly independent vectors and $\varangle(v, w)=\alpha$. We assume for simplicity that $0<\alpha \leq \pi / 2$ and $\|v\| \geq\|w\|$. We define an equivalence relation $\sim$ on $\mathbb{R}^{2}$ by declaring that $x \sim y$ (for $x, y \in \mathbb{R}^{2}$ ) iff there exist integers $k, l$ such that $x=y+k \cdot v+l \cdot w$. Then $T_{\alpha}(v, w)=\mathbb{R}^{2} / \sim$ with the quotient topology is a smooth closed oriented 2-dimensional differentiable manifold.

DeFinition 3.4. $T_{\alpha}(v, w)$ equipped with the Riemannian metric induced from the canonical metric on $\mathbb{R}^{2}$ is called a slanted torus here.

Let $\mathscr{F}$ be the family of all nontaut codimension one oriented foliations on $T_{\alpha}(v, w)$ and

$$
I(\mathcal{F})=\int_{T_{\alpha}(v, w)}\left|H_{\mathcal{F}}\right|^{2} \Omega
$$

for $\mathcal{F} \in \mathscr{F}$. We will show that

$$
I(\mathcal{F})>2 \cdot I\left(T_{\alpha}(v, w)\right)
$$

for any foliation $\mathcal{F} \in \mathscr{F}$.

First, we should compute the isoperimetric constant $I\left(T_{\alpha}(v, w)\right)$. Since a one-dimensional submanifold of $T_{\alpha}(v, w)$ which divides $T_{\alpha}(v, w)$ into two open submanifolds and has shortest boundary is either a union of two closed geodesics each of length $\|w\|$ or a circle, we get

$$
I\left(T_{\alpha}(v, w)\right)= \begin{cases}4 \pi & \text { when } \pi\|v\| \sin \alpha<2\|w\|, \\ \frac{8\|w\|}{\|v\| \sin \alpha} & \text { when } \pi\|v\| \sin \alpha \geq 2\|w\| .\end{cases}
$$

Theorem 3.5. For any $\mathcal{F} \in \mathscr{F}$,

$$
I(\mathcal{F})>2 \cdot I\left(T_{\alpha}(v, w)\right) .
$$

Moreover, if $\pi\|v\| \sin \alpha \geq 2\|w\|$, then

$$
\inf _{\mathcal{F} \in \mathscr{F}} I(\mathcal{F})=2 \cdot I\left(T_{\alpha}(v, w)\right) .
$$


Proof. Let $\mathcal{F}$ be a nontaut dimension one foliation on $T_{\alpha}(v, w)$. Then $\mathcal{F}$ has nondense leaves. Hence, by Kneser's theorem [Kn] there exist saturated subsets $A_{i} \subset T_{\alpha}(v, w), i=1, \ldots, \infty$, such that:

(1) $T_{\alpha}(v, w)=\bigcup_{i=1}^{\infty} A_{i}$

(2) for any $i=1, \ldots, \infty$ the set $A_{i}$ is an annulus, that is, $A_{i}$ is connected and its boundary $\partial A_{i}$ is a union of two closed curves which are compact leaves of $\mathcal{F}$ from a nonzero homotopy class,

(3) for any $i, j=1, \ldots, \infty$ connected components of $\partial A_{i}$ are homotopy equivalent to components of $\partial A_{j}$,

(4) the annuli $A_{i}$ are foliated in one of the following ways:

(a) all the leaves of $\mathcal{F}$ in $A_{i}$ are compact, hence they are homotopy equivalent to components of $\partial A_{i}$,

(b) all the leaves of $\mathcal{F}$ contained in $A_{i}$ tend in a spiral way to components of $\partial A_{i}$ which are oriented in this same way,

(c) all the leaves of $\mathcal{F}$ contained in $A_{i}$ approach the components of $\partial A_{i}$ which are oriented in the opposite way (Reeb components).

It is easy to see that the number $k_{\mathcal{F}}$ of minimal positively or negatively foliated domains of $T_{\alpha}(v, w)$ is equal to the number of Reeb components of $\mathcal{F}$. Moreover $k_{\mathcal{F}}$ is a positive even integer.

Let $A$ be a set of type (4.c), that is, a Reeb component. Then, since $\left.H_{\mathcal{F}}\right|_{A}$ is not a constant function, by 3.2 we have

$$
\int_{A}\left|H_{\mathcal{F}}\right|^{2} \Omega>\frac{(\text { Length } \partial A)^{2}}{\text { Area } A} .
$$

Since the number of Reeb components of $\mathcal{F}$ is at least 2, there are two distinct Reeb components $A, B \subset T_{\alpha}(v, w)$ and

$$
\begin{aligned}
\int_{T_{\alpha}(v, w)}\left|H_{\mathcal{F}}\right|^{2} \Omega & \geq \int_{A \cup B}\left|H_{\mathcal{F}}\right|^{2} \Omega>\frac{(\text { Length } \partial A)^{2}}{\text { Area } A}+\frac{(\text { Length } \partial B)^{2}}{\text { Area } B} \\
& \geq 4 l^{2}\left(\frac{1}{\text { Area } A}+\frac{1}{\text { Area } B}\right) \geq 4 l^{2}\left(\frac{1}{\text { Area } A}+\frac{1}{T-\text { Area } A}\right) \\
& \geq 4 l^{2} \inf _{a \in(0, T)}\left(\frac{1}{a}+\frac{1}{T-a}\right)=4 l^{2}\left(\frac{2}{T}+\frac{2}{T}\right)=\frac{16 l^{2}}{T} \\
& \geq \frac{16\|w\|^{2}}{\|v\|\|w\| \sin \alpha} \geq 2 \cdot I\left(T_{\alpha}(v, w)\right)
\end{aligned}
$$

where $T=$ Area $T_{\alpha}(v, w)$ and $l$ is the length of the shortest component of $\partial A \cup \partial B$. We have thus shown (18).

Let now $\alpha, v$ and $w$ be such that $\pi\|v\| \sin \alpha \geq 2\|w\|$. We can assume that $v=(\|v\| \sin \alpha,\|v\| \cos \alpha)$ and $w=(0,\|w\|)$. Let $r=\frac{1}{4}\|v\| \sin \alpha$. For any 
$\varepsilon$ from the interval $(0, r]$ we will construct a foliation $\mathcal{F}_{\varepsilon} \in \mathscr{F}$ such that

$$
I\left(\mathcal{F}_{\varepsilon}\right) \leq 2 \cdot I\left(T_{\alpha}(v, w)\right)+\delta(\varepsilon)
$$

where $\delta(\varepsilon)>0$ and $\lim _{\varepsilon \rightarrow 0} \delta(\varepsilon)=0$.

We first define $f_{\varepsilon}:(0, r] \rightarrow \mathbb{R}$ by

$$
f_{\varepsilon}=f_{1} \cdot f_{2}^{\varepsilon},
$$

where

$$
\begin{aligned}
& f_{1}(x)=-\sqrt{r^{2}-(x-r)^{2}}, \\
& f_{2}^{\varepsilon}(x)= \begin{cases}1-\frac{1}{x} e^{1 /(x-\varepsilon)} & \text { for } x<\varepsilon, \\
1 & \text { for } x \geq \varepsilon .\end{cases}
\end{aligned}
$$

The function $f_{\varepsilon}$ is smooth and has the following properties:

- $\lim _{x \rightarrow 0^{+}} f_{\varepsilon}(x)=+\infty$,

- the graph of $f_{\varepsilon}$ over $(\varepsilon, r]$ is a piece of the circle with center $(r, 0)$ and radius $r$.

Let $k_{\varepsilon}(x)$ be the curvature of the graph of $f_{\varepsilon}$ at $\left(x, f_{\varepsilon}(x)\right)$. Then $k_{\varepsilon}$ : $(0, r] \rightarrow \mathbb{R}$ is a smooth function with the following properties:

- $\lim _{x \rightarrow \varepsilon} k_{\varepsilon}(x)=1 / r$

- $\left.k_{\varepsilon}\right|_{[\varepsilon, r]}=1 / r$

- $\lim _{x \rightarrow 0^{+}} k_{\varepsilon}(x)=0$ because $f_{\varepsilon}$ has a vertical asymptote $x=0$.

Moreover, the function $F: D_{F}=(0, r] \times(0, r] \rightarrow \mathbb{R}$ defined by $F(\varepsilon, x)=$ $k_{\varepsilon}(x)$ is smooth, hence bounded on $D_{F}$. We put $B=\sup \left\{F(p) ; p \in D_{F}\right\}<\infty$.

Let $\widetilde{f}_{\varepsilon}:(0,2 r) \rightarrow \mathbb{R}$ be defined by

$$
\widetilde{f}_{\varepsilon}(x)= \begin{cases}f_{\varepsilon}(x) & \text { for } 0<x \leq r \\ f_{\varepsilon}(2 r-x) & \text { for } r<x<2 r .\end{cases}
$$

It is easy to verify that $\widetilde{f}_{\varepsilon}$ is smooth. Moreover, its graph is symmetric about the line $x=r$. We denote by $W_{\varepsilon}^{c}$ the graph of $\widetilde{f}_{\varepsilon}+c$ where $c \in \mathbb{R}$. The one-parameter family $\left\{W_{\varepsilon}^{c}\right\}_{c \in \mathbb{R}}$ defines a one-dimensional foliation of $(0,2 r) \times \mathbb{R}$ invariant under vertical translations. Similarly, we construct a foliation on $(2 r, 4 r) \times \mathbb{R}$. As a result we get a one-dimensional foliation of $[0,4 r] \times \mathbb{R}$ by completing the previous one with the lines $x=0, x=2 r$ and $x=4 r$. Because this foliation is invariant under vertical translations, it determines a foliation $\mathcal{F}_{\varepsilon} \in \mathscr{F}$.

Therefore, we have

$$
\left|H_{\mathcal{F}_{\varepsilon}}\left([(x, y)]_{\sim}\right)\right|= \begin{cases}\left|k_{\varepsilon}(x)\right| & \text { for } x \in(0,4 r) \backslash\{2 r\} \\ 0 & \text { for } x \in\{0,2 r, 4 r\}\end{cases}
$$


where $0 \leq t \leq 1$ and $(x, y)=t v+(1-t) w$. Moreover, we get

$$
\begin{aligned}
I\left(\mathcal{F}_{\varepsilon}\right) & =\int_{T_{\alpha}(v, w)}\left|H_{\mathcal{F}_{\varepsilon}}\right|^{2} \Omega=4 \int_{0}^{r} \int_{x \operatorname{ctg} \alpha}^{x \operatorname{ctg} \alpha+\|w\|} k_{\varepsilon}(x)^{2} d y d x \\
& =4\|w\| \int_{0}^{r} k_{\varepsilon}(x)^{2} d x=4\|w\| \int_{0}^{\varepsilon} k_{\varepsilon}(x)^{2} d x+4\|w\| \int_{\varepsilon}^{r} k_{\varepsilon}(x)^{2} d x \\
& \leq 4\|w\| \varepsilon B^{2}+4\|w\|(r-\varepsilon) \frac{1}{r^{2}} \leq 4\|w\| \varepsilon B^{2}+4\|w\| \frac{1}{r} \\
& =4\|w\| \varepsilon B^{2}+\frac{16\|w\|}{\|v\| \sin \alpha}=4\|w\| \varepsilon B^{2}+2 \cdot I\left(T_{\alpha}(v, w) .\right.
\end{aligned}
$$

Then, putting $\delta(\varepsilon)=4\|w\| \varepsilon B^{2}$ we obtain

$$
\lim _{\varepsilon \rightarrow 0} \delta(\varepsilon)=0
$$

hence $\inf _{\mathcal{F} \in \mathscr{F}} I(\mathcal{F})=2 \cdot I\left(T_{\alpha}(v, w)\right)$. This ends the proof of Theorem 3.5.

Let $\mathscr{T}$ be the family of all one-dimensional submanifolds $C$ of $T_{\alpha}(v, w)$ dividing the slanted torus $T_{\alpha}(v, w)$ into two open submanifolds $M_{1}$ and $M_{2}$ of genus 1 . We define a new isoperimetric constant as follows:

$$
I_{1}\left(T_{\alpha}(v, w)\right)=\inf _{C \in \mathscr{T}} \frac{(\text { Length } C)^{2}}{\min \left\{\text { Area } M_{1}, \text { Area } M_{2}\right\}} .
$$

Then the reasoning in the proof of Theorem 3.5 yields immediately the following corollary.

Corollary 3.6. For any nontaut one-dimensional oriented foliation $\mathcal{F} \in \mathscr{F}$ of $T_{\alpha}(v, w)$ we have

$$
I(\mathcal{F})>2 \cdot I_{1}\left(T_{\alpha}(v, w)\right)
$$

Moreover

$$
\inf _{\mathcal{F} \in \mathscr{F}} I(\mathcal{F})=I_{1}\left(T_{\alpha}(v, w)\right)
$$

Corollary 3.7. For any nontaut one-dimensional oriented foliation $\mathcal{F}$ of the standard flat torus $T^{2}=T_{\pi / 2}(v, v)$ (where $\left.\|v\|=1\right)$ we have

$$
I(\mathcal{F})>2 \cdot I\left(T^{2}\right)
$$

3.3. Sphere $S^{3}$. We now consider the three-dimensional sphere $S^{3}=$ $\left\{x \in \mathbb{R}^{4} ;\|x\|=1\right\}$ with the Riemannian metric induced from the canonical metric on $\mathbb{R}^{4}$. We denote by $\mathscr{M}$ the family of all closed codimension one genus one submanifolds $T$ of $S^{3}$. Then any $T \in \mathscr{M}$ is a torus separating $S^{3}$ into two open connected submanifolds $M_{1}$ and $M_{2}$. We define a new isoperimetric constant $I_{1}\left(S^{3}\right)$ by

$$
I_{1}\left(S^{3}\right)=\inf _{T \in \mathscr{M}} \frac{(\text { Area } T)^{3}}{\min \left\{\operatorname{Vol} M_{1}, \operatorname{Vol} M_{2}\right\}^{2}} .
$$


Obviously $I_{1}\left(S^{3}\right) \geq I\left(S^{3}\right)$. Let now $\mathscr{F}$ be the family of all nontaut transversely oriented codimension one foliations on $S^{3}$. We will show that

$$
I(\mathcal{F})>2 \cdot I_{1}\left(S^{3}\right)
$$

for any $\mathcal{F} \in \mathscr{F}$.

First of all we will estimate $I_{1}\left(S^{3}\right)$. The volume of $S^{3}$ equals $2 \pi^{2}$. Note that the sets

$$
\begin{aligned}
& A_{1}^{r}=\left\{\left(x_{1}, x_{2}, x_{3}, x_{4}\right) \in S^{3} ; x_{1}^{2}+x_{2}^{2}<r^{2} \wedge x_{3}^{2}+x_{4}^{2}>1-r^{2}\right\}, \\
& A_{2}^{r}=\left\{\left(x_{1}, x_{2}, x_{3}, x_{4}\right) \in S^{3} ; x_{1}^{2}+x_{2}^{2}>r^{2} \wedge x_{3}^{2}+x_{4}^{2}<1-r^{2}\right\}
\end{aligned}
$$

are open submanifolds of $S^{3}$ and $\partial A_{1}^{r}=\partial A_{2}^{r}=T_{r}$, where

$$
T_{r}=\left\{\left(x_{1}, x_{2}, x_{3}, x_{4}\right) \in S^{3} ; x_{1}^{2}+x_{2}^{2}=r^{2} \wedge x_{3}^{2}+x_{4}^{2}=1-r^{2}\right\}
$$

is a torus contained in $S^{3}$. Moreover $A_{1}^{r} \cup A_{2}^{r} \cup T_{r}=S^{3}$, hence $T_{r} \in \mathscr{M}$. Of course, Area $T_{r}=4 \pi^{2} r \sqrt{1-r^{2}}$. It is easy to see that

$$
\operatorname{Vol} A_{1}^{r}=2 \pi^{2} r^{2}, \quad \operatorname{Vol} A_{2}^{r}=2 \pi^{2}\left(1-r^{2}\right) .
$$

Therefore

$$
\inf _{r \in(0,1)} \frac{\left(\text { Area } T_{r}\right)^{3}}{\min \left\{\operatorname{Vol} A_{1}^{r}, \operatorname{Vol} A_{2}^{r}\right\}^{2}}=\inf _{r \in(0,1)} \frac{\left(4 \pi^{2} r \sqrt{1-r^{2}}\right)^{3}}{\min \left\{2 \pi^{2} r^{2}, 2 \pi^{2}\left(1-r^{2}\right)\right\}^{2}}=8 \pi^{2},
$$

hence $I_{1}\left(S^{3}\right) \leq 8 \pi^{2}$. Determining the constant $I_{1}\left(S^{3}\right)$ is not easy and is connected with the so-called Lawson conjecture [Law] which says that any minimal torus in $S^{3}$ is isometric to the Clifford torus $T_{1 / \sqrt{2}}$.

Let $H_{r}$ and $K_{r}$ be respectively the mean curvature function and the Gauss-Kronecker curvature of $T_{r}$ considered as a submanifold of $S^{3}$. An easy computation shows that

$$
\begin{aligned}
\left|H_{r}(x)\right| & =\frac{\left|1-2 r^{2}\right|}{r \sqrt{1-r^{2}}}, \\
K_{r}(x) & =-1,
\end{aligned}
$$

for any $x \in T_{r}$. (Hence the Clifford torus $T_{1 / \sqrt{2}}$ is a minimal submanifold of $S^{3}$.)

We denote by $H_{\mathcal{F}}$ and $K_{\mathcal{F}}$ respectively the mean curvature function and the Gauss-Kronecker curvature of the leaves of $\mathcal{F} \in \mathscr{F}$. Obviously,

$$
H_{\mathcal{F}}^{2} \geq 2\left|K_{\mathcal{F}}\right|+2 K_{\mathcal{F}}
$$

THEOREM 3.8. Let $\mathcal{F}$ be a transversely oriented codimension one foliation on $S^{3}$. Then

$$
I(\mathcal{F})=\int_{S^{3}}\left|H_{\mathcal{F}}\right|^{3} \Omega>16 \pi^{2}
$$

\section{Moreover}

$$
\inf _{\mathcal{F} \in \mathscr{F}} I(\mathcal{F})=16 \pi^{2}
$$


Proof. By the Novikov theorem, any foliation $\mathcal{F}$ has a Reeb component, hence is nontaut.

By (32) and Theorems 2.8 and 2.9 we have

$$
\int_{S^{3}} H_{\mathcal{F}}^{2} \Omega \geq 2\left(\int_{S^{3}}\left|K_{\mathcal{F}}\right| \Omega+\int_{S^{3}} K_{\mathcal{F}} \Omega\right)>2\left(2 \pi^{2}+2 \pi^{2}\right)=8 \pi^{2} .
$$

Moreover, by Hölder's inequality we get

$$
\int_{S^{3}} H_{\mathcal{F}}^{2} \Omega \leq\left(\int_{S^{3}}\left(H_{\mathcal{F}}^{2}\right)^{3 / 2}\right)^{2 / 3}\left(\int_{S^{3}} \Omega\right)^{1 / 3}
$$

hence

$$
I(\mathcal{F})=\int_{S^{3}}\left|H_{\mathcal{F}}\right|^{3} \Omega \geq\left(\operatorname{Vol} S^{3}\right)^{-1 / 2}\left(\int_{S^{3}} H_{\mathcal{F}}^{2} \Omega\right)^{3 / 2}>\frac{1}{\pi \sqrt{2}}\left(8 \pi^{2}\right)^{3 / 2}=16 \pi^{2} .
$$

We have thus shown (33).

Now we put $r=1 / \sqrt{2}$ and consider $A_{1}^{r}$ and $A_{2}^{r}$. Let $0<\varepsilon \leq r$. Similarly to the case of slanted tori we will construct a foliation $\mathcal{F}_{\varepsilon} \in \mathscr{F}$ such that

$$
I\left(\mathcal{F}_{\varepsilon}\right) \leq 16 \pi^{2}+\delta(\varepsilon),
$$

where $\delta(\varepsilon)>0$ and $\lim _{\varepsilon \rightarrow 0} \delta(\varepsilon)=0$. For each $\varepsilon$ the sphere $S^{3}$ will be divided into two saturated subsets $A_{1}^{r}$ and $A_{2}^{r}$ and a compact leaf $T_{r}$. We will only describe the foliation on $A_{1}^{r}$ (the construction on $A_{2}^{r}$ is analogous).

We put $D_{r}=\left\{x \in \mathbb{R}^{2} ;\|x\| \leq r\right\}$. Let $\psi: D_{r} \times \mathbb{R} \rightarrow \overline{A_{1}^{r}}$ be given by

$$
\psi\left(x_{1}, x_{2}, y\right)=\left(x_{1}, x_{2}, \sqrt{1-\|x\|^{2}} \cos y, \sqrt{1-\|x\|^{2}} \sin y\right),
$$

where $x=\left(x_{1}, x_{2}\right)$ and $\left(x_{1}, x_{2}, y\right) \in D_{r} \times \mathbb{R}$. It is easy to see that $\psi\left(D_{r} \times \mathbb{R}\right)$ $=\overline{A_{1}^{r}}$ and that $\psi$ is a covering map, because $\left.\psi\right|_{D_{r} \times(a, b)}$ is a diffeomorphism for any $a, b \in \mathbb{R}$ such that $b-a<2 \pi$. We put $S_{p}^{c}=\left\{x \in S^{3} ; x \cdot p=c\right\}$ for $p \in S^{3}$ and $0 \leq c<1$. The set $S_{p}^{c}$ is a two-dimensional sphere of radius $\sqrt{1-c^{2}}$ contained in $S^{3}$. The mean curvature function of $S_{p}^{c}$ is constant and its absolute value equals $2 c / \sqrt{1-c^{2}}$.

We note that

$$
\overline{A_{1}^{r}}=\bigcup_{p \in C} S_{p}^{r},
$$

where $C=\left\{\left(x_{1}, x_{2}, x_{3}, x_{4}\right) \in S^{3} ; x_{1}=0 \wedge x_{2}=0 \wedge x_{3}^{2}+x_{4}^{2}=1\right\}$. Indeed, first we will show that $S_{p}^{r} \subset \overline{A_{1}^{r}}$ for any $p \in C$. Let $x \in S_{p}^{r}, v=\left(x_{3}, x_{4}\right)$ and $w=\left(p_{3}, p_{4}\right)$. Then $|v|^{2}=|v|^{2}|w|^{2} \geq(v \cdot w)^{2}=1 / 2$, hence $x_{3}^{2}+x_{4}^{2} \geq 1 / 2$ and $x \in \overline{A_{1}^{r}}$. Let now $x \in \overline{A_{1}^{r}}$ and $\left(x_{3}, x_{4}\right)=R(\cos \alpha, \sin \alpha)$, where $R^{2} \geq 1 / 2$. Then there exists $\beta \in \mathbb{R}$ such that $\cos (\beta-\alpha)=1 /(R \sqrt{2})$, hence $x \in S_{p}^{r}$ for $p=(0,0, \cos \beta, \sin \beta)$. 
Moreover we observe that for any $p \in C$ the set $\psi^{-1}\left(S_{p}^{r}\right)$ is a countable union of surfaces of revolution. Indeed, for $p=(0,0, \cos \beta, \sin \beta)$ we see that

$$
(x, y) \in \psi^{-1}\left(S_{p}^{r}\right) \quad \text { iff } \quad \cos (y-\beta)=\left(2\left(1-\|x\|^{2}\right)\right)^{-1 / 2},
$$

hence for any $x \in D_{r}$ with $\|x\|=R$ the equality $\cos (y-\beta)=\left(2\left(1-R^{2}\right)\right)^{-1 / 2}$ holds iff $y=y_{0}+2 k \pi$ or $y=-y_{0}+2 k \pi$ for some $k \in \mathbb{Z}$, where $y_{0}$ is the unique real number such that $\cos \left(y_{0}-\beta\right)=\left(2\left(1-R^{2}\right)\right)^{-1 / 2}$ and $\beta \leq y_{0} \leq \beta+\pi$. The set $\psi^{-1}\left(S_{p}^{r}\right) \cap\left(\left\{x \in D_{r} ;\|x\|=R\right\} \times \mathbb{R}\right)$ is thus a countable union of horizontal circles of radius $R$. Moreover,

$$
\psi^{-1}\left(S_{p}^{r}\right)=\bigcup_{k \in \mathbb{Z}}\left\{(x, y) \in D_{r} \times I_{k} ; \cos (y-\beta)=\left(2\left(1-\|x\|^{2}\right)\right)^{-1 / 2}\right\},
$$

where $I_{k}=[\beta-\pi / 4+2 k \pi, \beta+\pi / 4+2 k \pi]$. Our observation follows from the above and the fact that $\left.\psi\right|_{D_{r} \times(\beta-\pi / 4-\varepsilon, \beta+\pi / 4+\varepsilon)}$ is a diffeomorphism for $\varepsilon>0$ small enough.

Let $\varepsilon \leq r$. We put

$$
F_{\varepsilon}=F_{1} \cdot F_{2}^{\varepsilon},
$$

where $F_{1}:[0,1 / \sqrt{2}) \rightarrow[-\pi / 4,0]$ and $F_{2}^{\varepsilon}:[0,1 / \sqrt{2}) \rightarrow(-\infty, 1]$ are defined by

$$
\begin{aligned}
& F_{1}(\|x\|)=-\arccos \left(\frac{1}{\sqrt{2} \sqrt{1-\|x\|^{2}}}\right), \\
& F_{2}^{\varepsilon}(\|x\|)= \begin{cases}1+\frac{1}{\|x\|-r} e^{1 /(r-\varepsilon-\|x\|)} & \text { if }\|x\|>r-\varepsilon, \\
1 & \text { if }\|x\| \leq r-\varepsilon .\end{cases}
\end{aligned}
$$

Now, we rotate the graph of $F_{\varepsilon}$ around the line $\{0\} \times \mathbb{R}$ to obtain a surface of revolution. The family of the graphs of the functions $F_{\varepsilon} \circ\|\cdot\|+t, t \in \mathbb{R}$, is a two-dimensional foliation of the cylinder $D_{r} \times \mathbb{R}$ invariant under vertical translations. In this way we get a foliation on the set $A_{1}^{r}$ by lifting the latter foliation by the map $\psi$. Since $F_{2}^{\varepsilon}$ has analogous properties to those of $f_{2}^{\varepsilon}$ in (23), the foliation $\mathcal{F}_{\varepsilon}$ has the following properties:

- on $A_{1}^{r-\varepsilon}$, the leaves of $\mathcal{F}_{\varepsilon}$ are parts of spheres of radius $1 / \sqrt{2}$, hence $\left|H_{\mathcal{F}_{\varepsilon}}\right|_{A_{1}^{r-\varepsilon}} \mid=2$;

- each leaf of $\mathcal{F}_{\varepsilon}$ accumulates on the torus $T_{r}$;

- for any leaf $L \in \mathcal{F}_{\varepsilon}$ and a sequence $\left(x_{n}\right)_{n \in \mathbb{N}}$ of points of $L$ converging to some $x \in T_{r}$ we have $\lim _{n \rightarrow \infty}\left|H_{\mathcal{F}_{\varepsilon}}\left(x_{n}\right)\right|=0$;

- there exists a positive number $B$ such that $B \geq\left|H_{\mathcal{F}_{\varepsilon}}(x)\right|$ for any $x \in A_{1}^{r}$ and $0<\varepsilon \leq r$. 
We complete $\mathcal{F}_{\varepsilon}$ with the boundary leaf $T_{r}$. Then

$$
\begin{aligned}
I\left(\mathcal{F}_{\varepsilon}\right) & =\int_{S^{3}}\left|H_{\mathcal{F}_{\varepsilon}}\right|^{3} \Omega=2 \int_{A_{1}^{r}}\left|H_{\mathcal{F}_{\varepsilon}}\right|^{3} \Omega=2 \int_{A_{1}^{r-\varepsilon}}\left|H_{\mathcal{F}_{\varepsilon}}\right|^{3} \Omega+2 \int_{A_{1}^{r} \backslash A_{1}^{r-\varepsilon}}\left|H_{\mathcal{F}_{\varepsilon}}\right|^{3} \Omega \\
& \leq 2 \cdot 8 \operatorname{Vol} A_{1}^{r-\varepsilon}+2 B^{3} \operatorname{Vol}\left(A_{1}^{r} \backslash A_{1}^{r-\varepsilon}\right) \\
& =16 \cdot 2 \pi^{2}(r-\varepsilon)^{2}+2 B^{3} \cdot 2 \pi^{2}\left(r^{2}-(r-\varepsilon)^{2}\right) \\
& \leq 16 \cdot 2 \pi^{2} r^{2}+4 \pi^{2} B^{3} \varepsilon(2 r-\varepsilon)=16 \pi^{2}+\delta(\varepsilon),
\end{aligned}
$$

where $\delta(\varepsilon)=4 \pi^{2} B^{3} \varepsilon(2 r-\varepsilon)>0$ and $\lim _{\varepsilon \rightarrow 0^{+}} \delta(\varepsilon)=0$. This ends the proof of (34) and of Theorem 3.8.

Corollary 3.9. For any transversely oriented codimension one foliation $\mathcal{F}$ on the sphere $S^{3}$ we have

$$
I(\mathcal{F})>2 \cdot I_{1}\left(S^{3}\right) \geq 2 \cdot I\left(S^{3}\right) .
$$

Equality (34) suggests that $I_{1}\left(S^{3}\right)=8 \pi^{2}$.

\section{References}

[CaLi] C. Camacho and A. Lins Neto, Geometric Theory of Foliations, Birkhäuser, Boston, MA, 1985.

[Ch] J. Cheeger, A lower bound for the smallest eigenvalue of the Laplacian, in: Problems in Analysis, Princeton Univ. Press, Princeton, NJ, 1970, 195-199.

[Ho] H. Hopf, Differential Geometry in the Large, Lecture Notes in Math. 1000, Springer, 1983.

[Kn] H. Kneser, Reguläre Kurvenscharen auf die Ringflächen, Math. Ann. 91 (1923), $135-154$.

[La] R. Langevin, Introduction to integral geometry, Notes of the Advanced Course on Integral Geometry, CRM, Bellaterra, 1999.

[La1] —, Feuilletages tendus, Bull. Soc. Math. France 107 (1979), 271-281.

[La2] - Courbure des feuilletages et surfaces (mesures et distributions de Gauss), thèse d'état, Orsay, 1980.

[LaLe] R. Langevin et G. Levitt, Courbure totale des feuilletages, Comment. Math. Helv. 57 (1982), 175-195.

[Law] H. B. Lawson, Lectures on Minimal Submanifolds, Vol. I, 2nd ed., Math. Lecture Ser., 9, Publish or Perish, Wilmington, DE, 1980.

[Mi] J. Milnor, Morse Theory, Princeton Univ. Press, Princeton, NJ, 1963.

[Os] G. I. Oshikiri, Tautness of codimension-one foliations and isoperimetric constants, Interdiscip. Inform. Sci. 1 (1994), 47-50.

[Re] G. Reeb, Sur la courbure moyenne des variétés intégrales d'une équation de Pfaff $\omega=0$, C. R. Acad. Sci. Paris 213 (1950), 101-102.

[Ru] H. Rummler, Quelques notions simples en géométrie riemannienne et leurs applications aux feuilletages compacts, Comment. Math. Helv. 54 (1979), 224-239.

[Sc] R. Schneider, Integral geometry-measure theoretic approach and stochastic applications, Notes of the Advanced Course on Integral Geometry, CRM, Bellaterra, 1999. 
[Su] D. Sullivan, A homological characterization of foliations consisting of minimal surfaces, Comment. Math. Helv. 54 (1979), 218-223.

Faculty of Mathematics

University of Łódź

Banacha 22

90-238 Łódź, Poland

E-mail: konradbl@imul.uni.lodz.pl

Reçu par la Rédaction le 4.4.2000

Révisé le 16.5.2001 